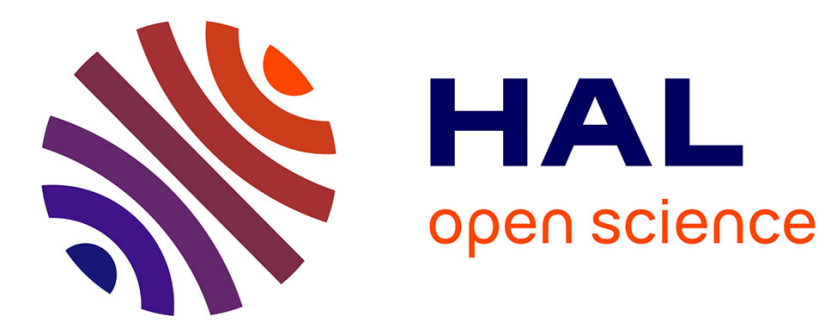

\title{
Serum N-Glycan Profile Shift During Human Ageing
}

Valerie Vanhooren, Sylviane Dewaele, Claude Libert, Sebastiaan Engelborghs, Peter Paul de Deyn, Olivier Toussaint, Florence Debacq-Chainiaux, Michel Poulain, Youri Glupczynski, Claudio Franceschi, et al.

\section{- To cite this version:}

Valerie Vanhooren, Sylviane Dewaele, Claude Libert, Sebastiaan Engelborghs, Peter Paul de Deyn, et al. Serum N-Glycan Profile Shift During Human Ageing. Experimental Gerontology, 2010, 45 (10), pp.738. 10.1016/j.exger.2010.08.009 . hal-00625923

\section{HAL Id: hal-00625923 \\ https://hal.science/hal-00625923}

Submitted on 23 Sep 2011

HAL is a multi-disciplinary open access archive for the deposit and dissemination of scientific research documents, whether they are published or not. The documents may come from teaching and research institutions in France or abroad, or from public or private research centers.
L'archive ouverte pluridisciplinaire HAL, est destinée au dépôt et à la diffusion de documents scientifiques de niveau recherche, publiés ou non, émanant des établissements d'enseignement et de recherche français ou étrangers, des laboratoires publics ou privés. 


\section{Accepted Manuscript}

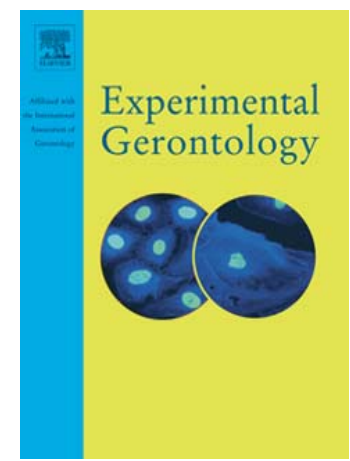

Serum N-Glycan Profile Shift During Human Ageing

Valerie Vanhooren, Sylviane Dewaele, Claude Libert, Sebastiaan Engelborghs, Peter Paul De Deyn, Olivier Toussaint, Florence Debacq-Chainiaux, Michel Poulain, Youri Glupczynski, Claudio Franceschi, Koos Jaspers, Ingrid van der Pluijm, Jan Hoeijmakers, Cuiying Chitty Chen

PII: $\quad$ S0531-5565(10)00234-2

DOI: $\quad$ doi: $10.1016 /$ j.exger.2010.08.009

Reference: $\quad$ EXG 8774

To appear in: $\quad$ Experimental Gerontology

Received date: 27 July 2010

Revised date: $\quad 13$ August 2010

Accepted date: 13 August 2010

Please cite this article as: Vanhooren, Valerie, Dewaele, Sylviane, Libert, Claude, Engelborghs, Sebastiaan, De Deyn, Peter Paul, Toussaint, Olivier, Debacq-Chainiaux, Florence, Poulain, Michel, Glupczynski, Youri, Franceschi, Claudio, Jaspers, Koos, der Pluijm, Ingrid van, Hoeijmakers, Jan, Chen, Cuiying Chitty, Serum NGlycan Profile Shift During Human Ageing, Experimental Gerontology (2010), doi: 10.1016/j.exger.2010.08.009

This is a PDF file of an unedited manuscript that has been accepted for publication. As a service to our customers we are providing this early version of the manuscript. The manuscript will undergo copyediting, typesetting, and review of the resulting proof before it is published in its final form. Please note that during the production process errors may be discovered which could affect the content, and all legal disclaimers that apply to the journal pertain. 
Vanhooren et al. Serum N-glycan biomarker

\section{Serum N-Glycan Profile Shift During Human Ageing}

Valerie Vanhooren $^{1,2}$, Sylviane Dewaele ${ }^{1,2}$, Claude Libert ${ }^{1,2}$, Sebastiaan Engelborghs ${ }^{3,4}$,

Peter Paul De Deyn ${ }^{3,4}$, Olivier Toussaint ${ }^{5}$, Florence Debacq-Chainiaux ${ }^{5}$, Michel Poulain ${ }^{6}$, Youri Glupczynski ${ }^{7}$, Claudio Franceschi ${ }^{8}$, Koos Jaspers ${ }^{9}$, Ingrid van der Pluijm ${ }^{9}$, Jan Hoeijmakers ${ }^{9}$ and Cuiying Chitty Chen ${ }^{1,2^{*}}$

${ }^{1}$ Department for Molecular Biomedical Research, VIB, Technologiepark 927, B-9052 GentZwijnaarde, Belgium

${ }^{2}$ Department of Biomedical Molecular Biology, Ghent University, Technologiepark 927, B9052 Gent-Zwijnaarde, Belgium

${ }^{3}$ Reference Center for Biological Markers of Memory Disorders, Laboratory of Neurochemistry and Behavior, and ${ }^{4}$ Biobank, Institute Born-Bunge, University of Antwerp, Universiteitsplein 1, B-2610 Antwerp, Belgium

${ }^{5}$ University of Namur - FUNDP / URBC 61, rue de Bruxelles, B-5000 Namur, Belgium ${ }^{6}$ GéDAP - Faculté des sciences économiques, sociales, politiques et de communication, UCL, Bd Devreux 6, B-6000 Charleroi, Belgium

${ }^{7}$ Cliniques universitaires UCL de Mont-Godinne 5530 Yvoir, Belgium

${ }^{8}$ Dept. Experimental Pathology, via S. Giacomo, 12 I-40126 Bologna, Italy

${ }^{9}$ DNage BV, Dept of Genetics, Erasmus University Medical Center, Rotterdam, the Netherlands

*Correspondence should be addressed to Cuiying Chitty Chen (Chitty@dmbr.UGent.be) Tel : +32 93313702; Fax: +3293313609

Key words: biomarker, ageing, age-related disease, $\mathrm{N}$-glycan profile 


\section{Abbreviations}

DSA-FACE: DNA sequencer-assisted, fluorophore-assisted carbohydrate

NGA2F: agalactosylated, core- $\alpha-1,6$-fucosylated biantennary

NA2F: bigalactosylated, core- $\alpha-1,6-$ fucosylated biantennary

CS: Cockayne Syndrome 


\begin{abstract}
Biomarkers indicating biological age are of significant interest for prevention, diagnosis and monitoring (and the treatment) of age-related diseases. We previously reported an alteration of serum N-glycan profile in old humans using "DNA Sequencer Adapted-Fluorophore Assisted Carbohydrate Electrophoresis" (DSA-FACE). To validate the shift in serum Nglycan profile during ageing, we studied serum N-glycan profiles in different age groups of healthy volunteers, patients with dementia, and patients with Cockayne Syndrome, a genetic DNA repair disorder involving neurodegeneration and premature ageing. We found that the $\log$ of the ratio of two glycans (NGA2F and NA2F), named GlycoAgeTest, remained steady up to the age of 40 years and thereafter gradually increased to reach its highest level in nonagenarians. Patients with dementia or Cockayne Syndrome had a higher GlycoAgeTest level than age-matched healthy individuals. We thus demonstrate that the value of GlycoAgeTest is better than chronological age for estimating the physiological age of a human individual, and that it could be used as an ageing biomarker for healthy humans. Our data indicate that the GlycoAgeTest could be used as a non-invasive surrogate marker for general health, for forecasting disease progression during ageing, and for monitoring the efficacy of anti-ageing food compounds.
\end{abstract}

\title{
1. Introduction
}

The ageing process, like many other biological processes, is regulated by classical signaling pathways and transcription factors. Many of these pathways were first discovered in small, short-lived organisms such as yeast, worms and flies, but a remarkable fraction of these pathways turn out to extend lifespan in mammals as well (Kenyon 2010). Lipoprotein metabolism, genomic instability, oxidative stress, cellular response to damage, inflammatory processes, TOR signaling, signals from the reproductive system, telomeres, insulin/IGF-1 
signaling and sirtuins have all been proposed as regulators of the ageing process (Whalley 2009, Kenyon 2010). For instance, DNA damage and repair plays a very important role in the ageing process. To avoid the deleterious consequences of genetic damage, the DNA of all organisms also encodes an intricate network of specialized DNA repair pathways. The vital importance of basic DNA repair mechanisms for maintaining genomic integrity is underscored by their extreme conservation from unicellular bacteria and yeast to man (Hoeijmakers 2001). Defects in DNA repair systems either cause embryonic death or result in severe pathology later in life. Many patients and mouse mutants with defects in different nucleotide excision repair factors exhibit phenotypes reminiscent of premature ageing. Defects in the transcription-coupled nucleotide excision repair factors CSA or CSB lead to Cockayne syndrome (CS) in humans (Mitchell 2003).

After middle age, the body starts to experience a decline in various functions, and this leads to a high risk for ageing-associated diseases, such as cardiovascular disease, cancer, arthritis, cataract, osteoporosis, type 2 diabetes, hypertension and Alzheimer's disease. The incidence of all of these diseases increases rapidly with ageing. These age-related diseases can accelerate the ageing process. Dementia encompasses deteriorations in several cognitive domains (Morris, 2005). It has been defined as a manifestation of deteriorating brain functions over time as a part of ageing due to death or functional decline of brain cells caused by neurodegeneration or other disease (Peng, 2003). Alzheimer's disease (AD), the most common type of dementia, is a progressive, degenerative brain disease accounting for 65$70 \%$ of all dementia cases (Blennow et al., 2006). The cause of AD is unknown, but ageing is one causative factor. The incidence of $\mathrm{AD}$ increases substantially after the age of 70 years and represents a major health concern and economic burden worldwide. Mutations that slow down ageing also postpone age-related diseases such as dementia (Kenyon 2010). 
The molecular mechanisms underlying the ageing process are only beginning to be unraveled, yet there is clear evidence that the rate of ageing differs substantially between members of the same species, including humans. In other words, "biological age" of an individual might be different from the chronological age, in which case the biological age should be a better indicator of health and life expectancy. Researchers are trying to identify age-related changes that gauge physical age and predict life span more accurately than by counting years. Due to the rapidly increasing average life expectancy in most developed countries, the prevalence of age-related diseases is increasing. This necessitates development of new effective strategies for prevention and early diagnosis of such conditions. As ageing in many species appears amenable to interventions, research into the process of ageing and means by which it can be slowed down has gained considerable momentum in recent years (Anisimov et al., 2010). To test new interventions such as drugs or food components, it is necessary to measure the effect of the intervention on the underlying process of ageing. Preferably, this should not be limited to the effect on one of the body's systems or functions, for example, on blood pressure or cholesterol levels, but should reveal whether the intervention slows down the ageing process itself. Currently, the only way to test interventions aimed at extending life in humans is to monitor people until the end of their lives to determine if life span has been affected.

Glycosylation is a form of co-translational and post-translational modification. Glycans serve a variety of structural and functional roles in membrane-bound and secreted proteins. An Nglycan (N-linked oligosaccharide) is a sugar chain covalently linked to an asparagine residue of a polypeptide chain. Analyses of protein sequence databases have revealed that about two thirds of those sequences are likely to be N-glycosylated (Varki A., 2009). Glycans can mediate a wide variety of biological functions by virtue of their mass, shape, charge and other physical properties. Unlike protein sequences, which are primary gene products, glycan chain structures are not encoded directly in the genome but are secondary gene products. Glycans 
are synthesized in many different combinations by a variety of competing and sequentially acting glycosidases and glycosyltransferases without use of a template (Varki A., 2009). Small alterations in the environment can cause dramatic changes in the glycans produced by a particular cell, and so N-glycosylation could mirror the status of the cell. We and others recently showed that the composition of serum $\mathrm{N}$-glycans changes during human ageing (Knezevic et al., 2010; Vanhooren et al., 2007). Certain relatively specific alterations in the expression of glycans are also found during oncogenic transformation and progression to malignancy, as well as in other pathological conditions, such as inflammation (Kramer and Schulz-Schaeffer, 2007). The spatial and temporal control of glycan expression implies the involvement of glycans in many physiological and pathological processes (Haltiwanger and Lowe, 2004; Ohtsubo and Marth, 2006). Since N-glycosylation reflects in part the state of the cell, it could be a suitable biomarker not only for healthy ageing but also for age-related disease.

To determine whether changes in the extent and nature of posttranslational modification of proteins could be hallmarks of biological age, we investigated the N-glycosylation status of proteins in human blood. We also examined the serum $\mathrm{N}$-glycosylation fingerprint in patients with age-related disease (dementia) and in patients suffering from Cockayne syndrome, a premature ageing syndrome caused by deficiency in a DNA repair. We defined a particular serum $\mathrm{N}$-glycan profile shift during ageing as a biomarker.

\section{Materials and methods}

\subsection{Serum samples from healthy Belgians}

Serum samples from 425 healthy Belgian adults were obtained from the Biobank, Institute Born-Bunge, and from the Transfusion Center of the Red Cross, Belgium, in accordance with Red Cross health standards. All of these people were in relatively good health and had no 
Vanhooren et al. Serum N-glycan biomarker

relevant acute or chronic disease affecting the immune system. They were divided in four age groups: $20-49(n=60), 50-75(n=160), 76-89(n=30)$ and 90-99 years $(n=175)$. Informed consent was obtained from all participants.

\subsection{Serum samples from healthy Italians}

Serum samples were obtained from 84 Italian centenarians (Vanhooren et al., 2007). All were in relatively good health and had no relevant acute or chronic disease affecting the immune system. As controls, we studied three groups of healthy Italian volunteers of different ages: 20-49 $(n=35), 50-75(n=37)$ and 76-99 years $(n=71)$. Informed consent was obtained from all participants.

\subsection{Serum samples from dementia patients}

Serum samples from 79 patients with autopsy-confirmed dementia were included. All sera samples were obtained from the Biobank, Institute Born-Bunge, Antwerp, Belgium. We recorded the scores of the Mini-Mental State Examination (MMSE) at the time of serum sampling, year of clinical diagnosis, clinical and pathological diagnosis, year of death, date of autopsy (Chen C, 2010). The study was approved by the local ethics committee (CME Middelheim Hospital, Antwerp).

\subsection{Serum samples from patients with Cockayne Syndrome (CS)}

Serum samples were obtained from six CS patients aged 3, 7, 8, 10, 10 and 20 years. Informed consent was obtained from parents or guardians. CS diagnosis was confirmed by functional Nucleotide excision repair (NER) assays in fibroblasts derived from these patients as previously described (Jaspers et al., 2007) 


\section{5. $N$-glycan fingerprinting}

$\mathrm{N}$-glycan fingerprinting of all the samples was done as detailed before (Vanhooren et al., 2008). N-glycan profiles were generated from 2- $\mu 1$ serum samples by DSA-FACE technology using a capillary electrophoresis (CE)-based ABI3130 Genetic Analyzer (Applied Biosystems, Foster City, CA, USA). The intra- and inter-assay coefficients of variation (CV) of the glycan analyses are less than $5 \%$.

\subsection{Statistical analysis}

All quantitative variables are presented as mean \pm SD. The unpaired t-test was used for comparison of two groups, and for comparison of more than two groups, one-way ANOVA was performed. All reported P-values are two-tailed, and P-values $<0.05$ are considered statistically significant. Statistical analysis was performed with SPSS15.0 software (SPSS, Chicago, IL, USA).

\section{Results}

\subsection{The healthy human ageing biomarker GlycoAgeTest: shift of the serum glycoprofile}

Our department developed an ultra-sensitive technique to quantitatively profile and sequence N-linked glycans using standard DNA-sequencing equipment (Laroy et al., 2006), and we adapted this technique for the study of human ageing (Vanhooren et al., 2009). Using "DNA Sequencer Adapted-Fluorophore Assisted Carbohydrate Electrophoresis" (DSAFACE), an accurate fingerprint of the glycan composition in serum or plasma can be obtained. The DSA-FACE procedure requires only $2 \mu 1$ of serum and involves labeling of N-glycans, profiling, and read-out (Fig. 1). A typical N-glycan profile of human serum and plasma was previously generated and the nine most prominent structures corresponding to glycans in the profile were identified previously (Liu et al., 2007). The sizes of the peaks, which represent 
Vanhooren et al. Serum N-glycan biomarker

the relative concentrations of the oligosaccharide structures, can be analyzed statistically to determine the correlation between specific glycans and ageing. This high-throughput technique is robust, reproducible, sensitive and quantitative.

Using this approach, we previously performed a thorough study of the N-glycosylation status of serum proteins during ageing in 100 healthy Belgian blood donors (Belgian Red Cross, Ghent, Belgium) (Vanhooren et al., 2007). The volunteers were from five age groups (20, 30, 40, 50 and 60 years), each consisting of 10 males and 10 females. Several glycan structures were found to be closely related to ageing. In particular, peak 1 (an agalactosylated core- $\alpha-1,6$-fucosylated biantennary; NGA2F) increased gradually with age, while peak 6 (a bigalactosylated, core- $\alpha-1,6$-fucosylated biantennary; NA2F) decreased (Fig. 2). The increases in peak 1 (agalacto-glycan, NGA2F) during ageing could be due to a greater abundance of beta-galactosidase, which removes two galactoses from Peak 6 (NA2F), or to a lesser abundance of beta-1,4-galactosyltransferase, which adds galactose to NGA2F (Fig 2). For precision and accuracy, a ratio of two variables is better than the use of a single measurement. In the current study, we defined the log ratio of NGA2F (peak1) and NA2F (peak6) as a potential ageing biomarker, named GlycoAgeTest $=\log [\mathrm{NGA}$ F/NA2F $]$ $(\log [\mathrm{P} 1 / \mathrm{P} 6])$. Using this formula, we reanalyzed its correlation with age in the Belgian volunteers. As shown in Figure 3, the level of GlycoAgeTest remained steady until 40 years, and then increased. This observation demonstrates that the N-glycan profile is rather stable and consistent up to the age of 40 years. It starts to increase by the age of 50 and even more so after the age of 60 years. The intra- and inter-assay coefficients of variations (CVs) of the glycan analysis are less than $5 \%$.

To see whether the shift in serum glycan profile is further increased at even higher later ages, we evaluated the GlycoAgeTest marker in 425 healthy Belgian volunteers in four age groups, including nonagenarians: 20-49 ( $\mathrm{n}=60), 50-75(\mathrm{n}=160), 76-89(\mathrm{n}=30)$ and 90-99 $(\mathrm{n}=175)$. 
Vanhooren et al. Serum N-glycan biomarker

Indeed, the GlycoAgeTest value gradually increased during ageing and reached the highest level in nonagenarians (90-99 years) (Fig 4).

To see whether the GlycoAgeTest marker is also applicable to other populations, we tested a group of 229 healthy Italian volunteers (female $n=160$, male $n=68$ ) of different ages: $20-49$ $(n=35), \quad 50-75 \quad(n=37), \quad 76-89 \quad(n=33), \quad 90-99 \quad(n=39)$ and $\geq 100$ years $(n=84)$. The GlycoAgeTest displayed the same trend of change with age observed in the Belgian volunteers (Fig. 4). This demonstrates that the alterations of glycan structures are closely related to the progress of human ageing at least in these two populations. Noteworthy, the level of GlycoAgeTest in the Italian volunteers was significantly lower $(\mathrm{p}<0.05)$ than in their age-matched Belgian.

\subsection{GlycoAgeTest in patients with dementia and patients with Cockayne syndrome}

We analyzed serum N-glycans by DSA-FACE in 79 Belgian patients with autopsy-confirmed dementia (31 of 50-75 years and 48 of 76-89 years). The GlycoAgeTest value was significantly higher $(\mathrm{p}<0.05)$ in dementia patients than in age-matched controls (Fig. 5), which suggests that the biological age of dementia patients corresponds to a higher chronological age.

On the other hand, progeroid syndromes can occur early in life and accelerate the ageing process, such as Cockayne syndrome (progeroid syndrome), which is a rare autosomal recessive disorder characterized by progressive multisystem degeneration and segmental premature ageing. The average reported life span of patients with CS is approximately 12.5 years (for extensive reviews on clinical characteristics of Cockayne syndrome: (Nance and Berry, 1992)(Licht et al., 2003). Because many of the traits are reminiscent of normal ageing, CS has been classified as a segmental premature ageing syndrome. 
Vanhooren et al. Serum N-glycan biomarker

To analyze the sensitivity of the GlycoAgeTest for a segmental progeroid syndrome, we determined the serum glycan profile in six CS patients (age of 3 to 17 years). As shown in figure 6, the level of GlycoAgeTest in the CS patients is highly heterogeneous, but even the lowest value is much higher than in the controls. The average score is in the same range as that of people in their nineties. Thus, even though the primary defect in CS is due to defective DNA damage repair, it appears that the profile of $\mathrm{N}$-glycans in serum of these patients corresponds with dramatic accelerated ageing.

\section{Discussion}

We profiled serum N-glycans by using DSA-FACE to validate our serum N-glycan ageing biomarker, GlycoAgeTest, in different age groups of healthy human volunteers, patients with dementia, and patients with CS, a genetic disease involving progressive neurodysfunction and many features of premature ageing. We demonstrate that the GlycoAgeTest is better than chronological age at estimating the physiological age of human and that it could be used as a biomarker of healthy ageing in humans.

As we age, the functions and physiological reactions of our body gradually slow down, and this becomes apparent mainly after middle age. We found that the level of our GlycoAgeTest in the two European nations (Belgium and Italy) remained unchanged until the age of 40 years, and then gradually increased with age. Interestingly, a lower serum GlycoAgeTest level was detected in the Italians than in age-matched Belgians. This discrepancy might hint a healthier ageing and longer life in Italians compared to Belgians. It has been reported by the EHEMU project (European Health Expectancies Monitoring Unit; Healthy Life Years In The European Union: Facts And Figures 2005) that the life expectancy at birth is slightly higher in Italy (78.2 years for men and 84 years for women) than Belgium (76.2 years for men and 81.9 for women) (http://ec.europa.eu/health/archive/ph_information/reporting/docs/hly_en.pdf). 
Vanhooren et al. Serum N-glycan biomarker

$\underline{\text { This difference could be partly due to common lifestyle between Belgium and Italy, and }}$ genetic polymorphisms (Melzer, 2008; Agundez et al., 1997; Melzer et al., 2007). It was recently documented that plasma $\mathrm{N}$-glycans is affected by age, body mass index, plasma lipid profiles and smoking (Knezevic et al., 2010). It is very interesting to further validate whether the GlycoAgeTest could identify accelerated ageing individual who is associated with lifestyle factors (such as smoking, obesity, lack of exercise and etc). The GlycoAgeTest is being validated further in 14 European nations in the context of the MARK-age project (www.mark-age.eu), a large-scale integrated project supported by the European Commission. Moreover, we found that the GlycoAgeTest value was high in patients with dementia compared to age-matched healthy volunteers, which indicates that the biological age of dementia patients is higher than their chronological age. This was also observed in CS. The fact that a defect in the mechanisms of DNA damage repair, and particularly those dealing with DNA lesions that block transcription can lead to a dramatic alteration in the serum Nglycan profile is very interesting. This implies that indirect links exist between defective functioning of specific DNA repair processes and the synthesis or turn-over of specific carbohydrate chains in the ER and Golgi apparatus. In any case, the findings are consistent with the idea that CS is a (segmental) premature ageing syndrome and that DNA damage repair is fundamental to various cellular and organismal processes, including $\mathrm{N}$-glycosylation. The one common factor between between dementia and CS is the ageing process, and this common association provides a strong argument that our GlycoAgeTest measures a process related to the ageing process.

We previously showed that $\mathrm{N}$-glycan markers can also clearly distinguish between the normal ageing process and disease because sugar structures are changed in different ways and to different extents. For instance, GlycoHCCTest and GlycoCirrhoTest are serum N-glycan diagnostic biomarkers for liver cancer and cirrhosis, respectively, and they were designed 
using different N-glycans from GlycoAgeTest (Callewaert et al., 2004; Liu et al., 2007). As the changes in $\mathrm{N}$-glycans are a consequence of genetic and environmental influences and physiologic responses, the GlycoAgeTest marker has a significant diagnostic potential. It could be used in combination with other existing ageing parameters: due to the intrinsic multicausal and multi-system nature of the ageing process, no single measurement has proven capable on its own of determining biological age (Barqawi et al., 2005; Johnson, 2006).

The American National Institute of Ageing (NIA) has proposed the following requirements for a biomarker of ageing (Ward et al., 2006). (1) It must predict the rate of ageing, i.e. it should determine how far one has progressed in his or her total life span, and it must predict this more accurately than chronological age. (2) It must monitor a basic process that underlies the ageing process, not the effects of disease. (3) Repeated testing should be harmless and should use, for example, a blood test or an imaging technique. (4) It should be possible to use it in humans and in laboratory animals, such as mice, so that it can be tested in lab animals before being validated in humans. Our GlycoAgeTest fulfills most of these criteria, and it is also extremely robust. Serum samples kept for years in the freezer yielded the same N-glycan profile even after numerous freeze-thaw cycles. Only $2 \mu 1$ of serum is needed for generating the $\mathrm{N}$-glycan profile, and so the marker can be easily repeated without harming the person. Moreover, this marker has already been validated in mice (unpublished data).

The mechanism behind these age-related $\mathrm{N}$-glycosylation alterations requires investigation. Preliminary data indicate that the N-glycosylation changes are not limited to a certain serum protein but cover all serum glycoproteins. This need further study. Whether the Nglycosylation alterations are caused by differences in biosynthesis or clearance or by other factors is currently under investigation and should provide us with many interesting answers about the mechanism behind our GlycoAgeTest. 
In summary, measuring the shift in serum N-glycan profile could be used to regularly evaluate the overall age-related health status. This not only facilitates the diagnosis of age-related diseases such as dementia, but also the follow-up of different therapies in individuals not suffering from accelerated ageing. As ageing is the biggest risk factor for all age-related diseases, which emerge mainly in middle age or later, detection of an abnormal N-glycan fingerprint and a faster-than-normal rate of ageing in a given individual would indicate the need for additional diagnostic and preventive measures. With a reliable GlycoAgeTest biomarker to predict biological age and monitor healthy ageing progress, it could become possible to take preventive measures to slow down ageing and age-related diseases.

\section{Acknowledgements}

We thank Dr. Amin Bredan for editing and Dr. Alexander Buerkle for critical reading the manuscript. This work was supported by a grant from Ghent University, Belgium (BOF No. 01106205), European commission FP6 Linkage (FP6-513866), European Commissionsponsored project Geha (Genetics of Healthy Ageing), (LSHM-CT-2004-503270), Markage (FP7-Health-2008- 200880), DNA Repair (LSHG-CT-2005-512113) and LifeSpan (LSHGCT-2007-036894), National Institute of Health (NIH)/National Institute of Ageing (NIA) (1PO1 AG-17242-02), NIEHS (1UO1 ES011044), and Netherlands Organization for Scientific Research (NWO) through the foundation of the Research Institute Diseases of the Elderly.

\section{Figure legends}

Fig. 1. A schematic overview of DNA Sequencer Adapted-Fluorophore Assisted Carbohydrate Electrophoresis (DSA-FACE) for monitoring the progression of human ageing. 
The GlycoTest model includes the following steps: (A) serum N-glycan labeling; (B) Nglycan profiling using DSA-FACE: the fingerprint with the nine most abundant desialylated N-glycans; and (C) analysis of GlycoAgeTest marker (log[P1/P6]). The structures of P1 (agalactosylated core- $\alpha-1,6$-fucosylated biantennary, NGA2F) and P6 (bigalactosylated core$\alpha-1,6$-fucosylated biantennary, NA2F) are shown. The symbols used in the structural formulas are $(\boldsymbol{\bullet}) \mathrm{N}$-acetylglucosamine (GlcNAc), $(\bullet)$ mannose, $(\circ)$ galactose, and $(\Delta)$ fucose.

Fig. 2. A serum N-glycan fingerprint generated by DSA-FACE of a young and an old Belgian healthy person (upper panel). P1 can be converted to P6 by $\beta-1,4-$ galactosyltransferase and P6 can be converted to P1 by $\beta$-galactosidase. The structures of P1 (agalactosylated core- $\alpha-1,6$-fucosylated biantennary, NGA2F) and P6 (bigalactosylated core$\alpha-1,6$-fucosylated biantennary, NA2F) are shown. The symbols used in the structural

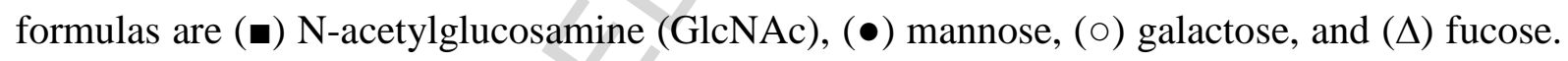
Arrow indicates the changed $\mathrm{N}$-glycan or peak in old age.

Fig. 3. The serum GlycoAgeTest values of healthy Belgian blood donors aged $\leq 60$ years. The level remains constant up to the age of 40 years and then gradually increases. The vertical axis represents the $95 \%$ confidence interval for the mean of the percentage of total N-glycan peak height. The statistical significance of differences between groups is indicated by the $\mathrm{p}$ value.

Fig. 4. The serum GlycoAgeTest values of a population of healthy Belgian (full lines) and Italian (dotted lines) volunteers of different ages: 20-49 (Belgian $n=60$, Italian $n=35$ ), 50-75 (Belgian $n=160$, Italian $n=37), 76-89$ (Belgian $n=30$, Italian $n=33$ ), 90-99 (Belgian $n=175$, Italian $n=39$ ) and $\geq 100$ years (only Italian $n=84$ ). The GlycoAgeTest level increases gradually with ageing. The vertical axis represents the $95 \%$ confidence interval for the mean of the percentage of total $\mathrm{N}$-glycan peak height. The statistical significance of differences between groups is shown by the $\mathrm{p}$ value. 
Fig. 5. The GlycoAgeTest performed on Belgian dementia patients of different ages: 50-75 $(n=30)$ and 76-99 years $(n=53)$. These levels were compared to Belgian healthy controls: $20-$ $49(n=60), 50-75(n=160), 76-89(n=30), 90-99$ (Belgian $n=175)$. The GlycoAgeTest value was higher in the dementia patients than in age-matched healthy controls. The vertical axis represents the $95 \%$ confidence interval for the mean of the percentage of total N-glycan peak height. The statistical significance of differences between groups is indicated by the p value.

Fig. 6. The GlycoAgeTest performed on patients with Cockayne Syndrome $(n=6)$. The GlycoAgeTest value was higher in these patients than in age-matched healthy controls. These values were compared to Belgian healthy controls: $20-49(n=60), 50-75 \quad(n=160), 76-89$ $(n=30), 90-99(n=175)$. The vertical axis represents the $95 \%$ confidence interval for the mean of the percentage of total $\mathrm{N}$-glycan peak height.

\section{References}

Morris, J.C., 2005. Dementia update 2005. Alzheimer disease and associated disorders 19, 100-17.

Peng, F.C., 2003. Is dementia a disease? Gerontology 49, 384-91.

Blennow, K., de Leon, M.J., Zetterberg, H., 2006. Alzheimer's disease. Lancet 368, 387-403. Anisimov, V.N., Zabezhinski, M.A., Popovich, I.G., Piskunova, T.S., Semenchenko, A.V., Tyndyk, M.L., Yurova, M.N., Antoch, M.P., Blagosklonny, M.V., 2010. Rapamycin extends maximal lifespan in cancer-prone mice. Am J Pathol 176, 2092-7.

Varki A., C.R., Esko J., Freeze H., Stanley P., Bertozzi C., Hart G., Etzler M., 2009. Essentials of Glycobiology. second edition.

Knezevic, A., Gornik, O., Polasek, O., Pucic, M., Redzic, I., Novokmet, M., Rudd, P.M., Wright, A.F., Campbell, H., Rudan, I., Lauc, G., 2010. Effects of aging, body mass index, plasma lipid profiles, and smoking on human plasma N-glycans. Glycobiology.

Vanhooren, V., Desmyter, L., Liu, X.E., Cardelli, M., Franceschi, C., Federico, A., Libert, C., Laroy, W., Dewaele, S., Contreras, R., Chen, C., 2007. N-glycomic changes in serum proteins during human aging. Rejuvenation Res 10, 521-531a.

Kramer, M.L., Schulz-Schaeffer, W.J., 2007. Presynaptic alpha-synuclein aggregates, not Lewy bodies, cause neurodegeneration in dementia with Lewy bodies. The Journal of neuroscience 27, 1405-10.

Haltiwanger, R.S., Lowe, J.B., 2004. Role of glycosylation in development. Annu Rev Biochem 73, 491-537. 
Ohtsubo, K., Marth, J.D., 2006. Glycosylation in cellular mechanisms of health and disease. Cell 126, 855-67.

Chen C, E.S., Dewaele S, Le Bastard N, Martin J, Vanhooren V, Libert C and De Deyn P., 2010. Altered serum glycomics in Alzheimer's disease: a potential blood biomarker? Rejuvenation Res.

Vanhooren, V., Laroy, W., Libert, C., Chen, C., 2008. N-Glycan profiling in the study of human aging. Biogerontology 9, 351-6.

Laroy, W., Contreras, R., Callewaert, N., 2006. Glycome mapping on DNA sequencing equipment. Nat Protoc 1, 397-405.

Vanhooren, V., Liu, X.E., Franceschi, C., Gao, C.F., Libert, C., Contreras, R., Chen, C., 2009. $\mathrm{N}$-glycan profiles as tools in diagnosis of hepatocellular carcinoma and prediction of healthy human ageing. Mech Ageing Dev 130, 92-7.

Liu, X.E., Desmyter, L., Gao, C.F., Laroy, W., Dewaele, S., Vanhooren, V., Wang, L., Zhuang, H., Callewaert, N., Libert, C., Contreras, R., Chen, C., 2007. N-glycomic changes in hepatocellular carcinoma patients with liver cirrhosis induced by hepatitis $\mathrm{B}$ virus. Hepatology 46, 1426-35.

Nance, M.A., Berry, S.A., 1992. Cockayne syndrome: review of 140 cases. Am J Med Genet 42, 68-84.

Licht, C.L., Stevnsner, T., Bohr, V.A., 2003. Cockayne syndrome group B cellular and biochemical functions. Am J Hum Genet 73, 1217-39.

Melzer, D., 2008. Genetic polymorphisms and human aging: association studies deliver. Rejuvenation Res 11, 523-6.

Agundez, J.A., Rodriguez, I., Olivera, M., Ladero, J.M., Garcia, M.A., Ribera, J.M., Benitez, J., 1997. CYP2D6, NAT2 and CYP2E1 genetic polymorphisms in nonagenarians. Age Ageing 26, 147-51.

Melzer, D., Hurst, A.J., Frayling, T., 2007. Genetic variation and human aging: progress and prospects. J Gerontol A Biol Sci Med Sci 62, 301-7.

Callewaert, N., Van Vlierberghe, H., Van Hecke, A., Laroy, W., Delanghe, J., Contreras, R., 2004. Noninvasive diagnosis of liver cirrhosis using DNA sequencer-based total serum protein glycomics. Nat Med 10, 429-34.

Barqawi, A.B., Golden, B.K., O'Donnell, C., Brawer, M.K., Crawford, E.D., 2005. Observed effect of age and body mass index on total and complexed PSA: analysis from a national screening program. Urology 65, 708-12.

Johnson, T.E., 2006. Recent results: biomarkers of aging. Exp Gerontol 41, 1243-6.

Ward, D.G., Cheng, Y., N'Kontchou, G., Thar, T.T., Barget, N., Wei, W., Billingham, L.J., Martin, A., Beaugrand, M., Johnson, P.J., 2006. Changes in the serum proteome associated with the development of hepatocellular carcinoma in hepatitis C-related cirrhosis. Br J Cancer 94, 287-92. 
A. N-glycan labeling
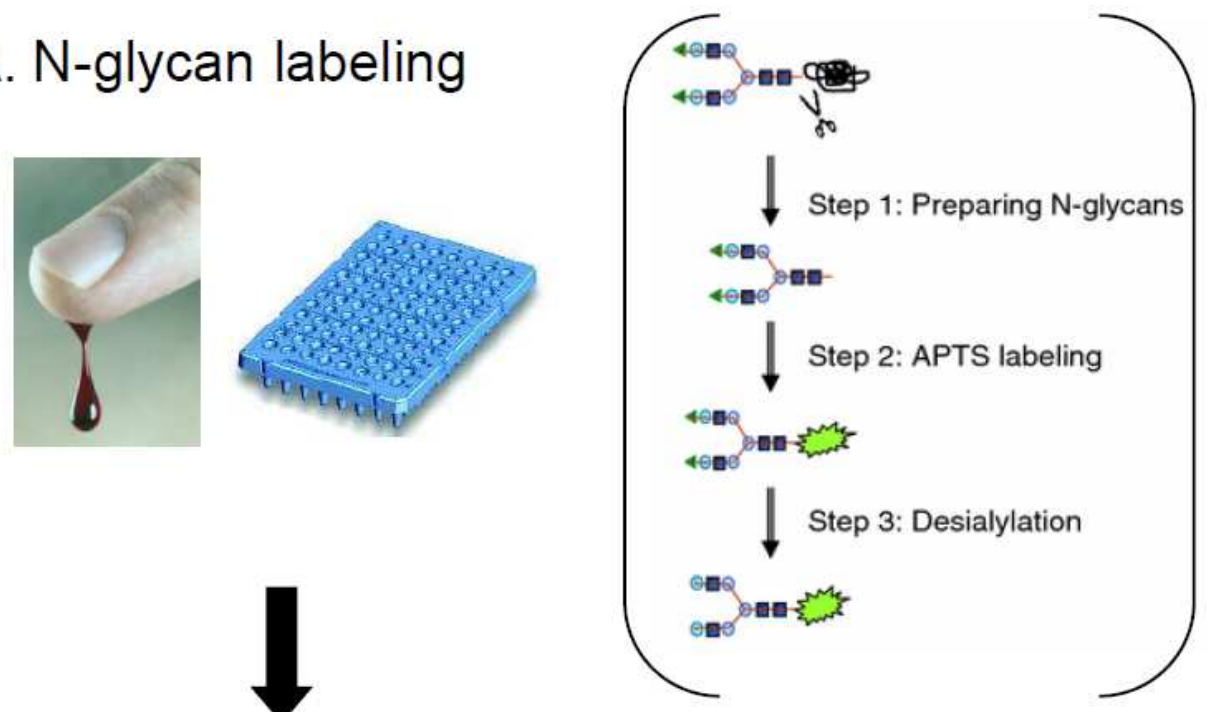

B. N-glycan profiling Using DNA-sequencer
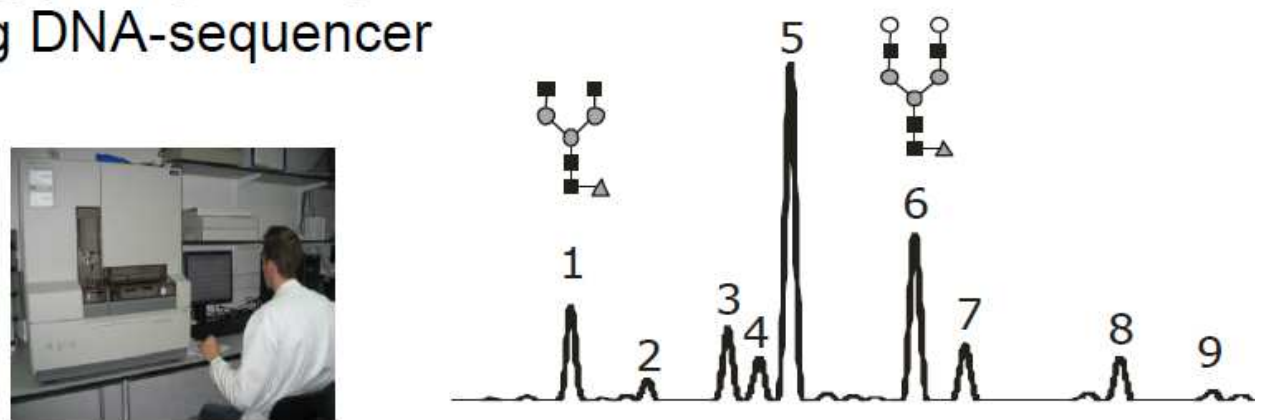

Figure 1

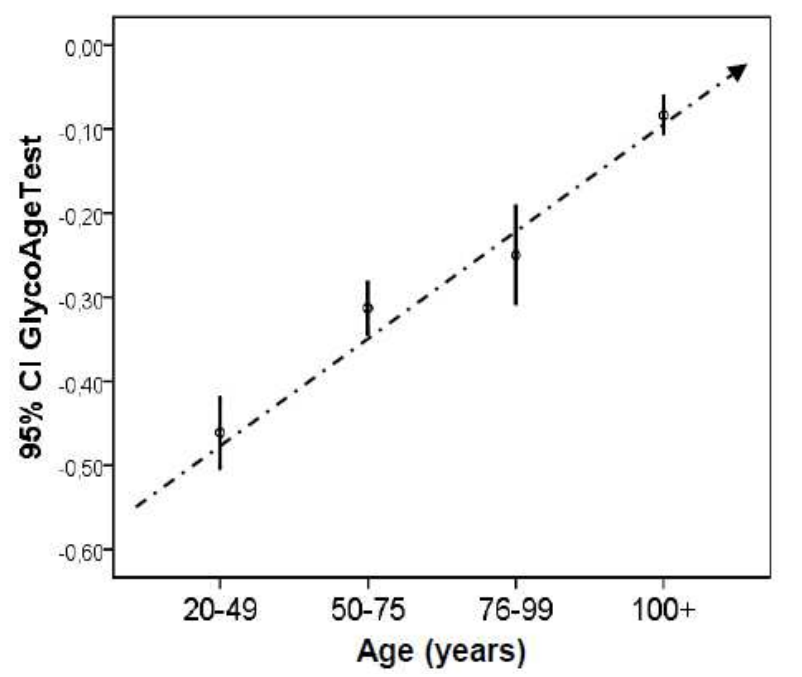




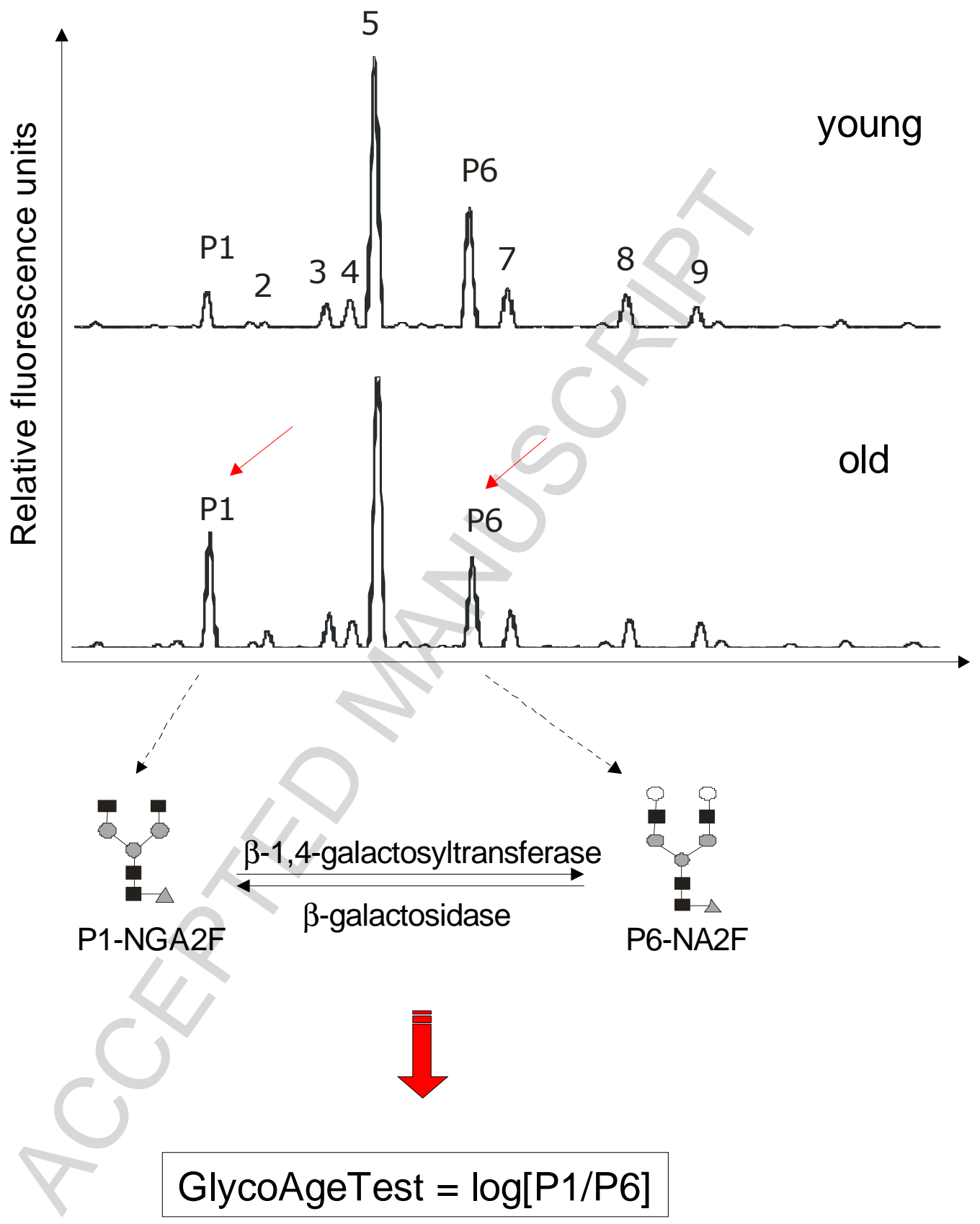

Figure 2 


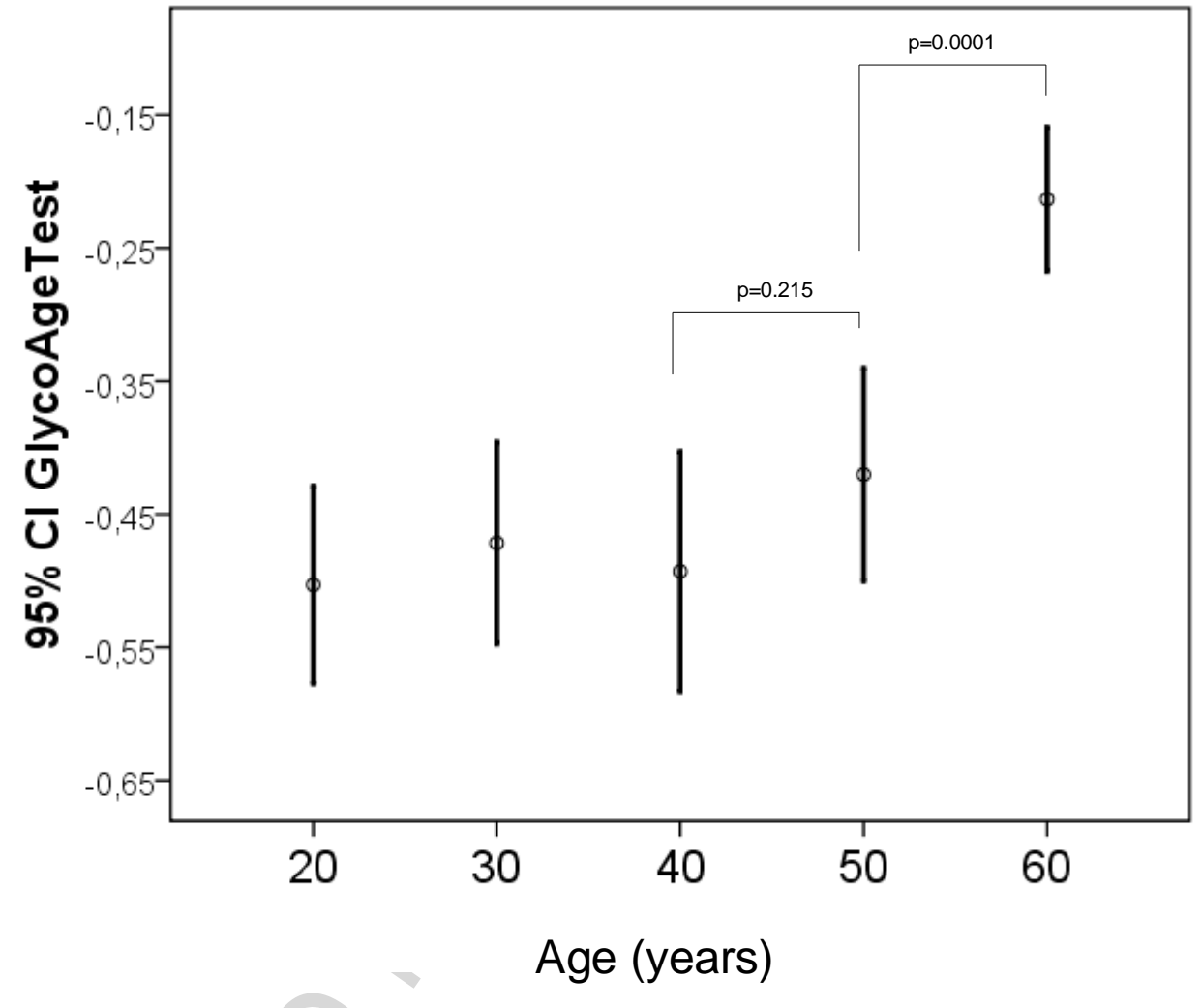

Figure 3 
Vanhooren et al. Serum N-glycan biomarker

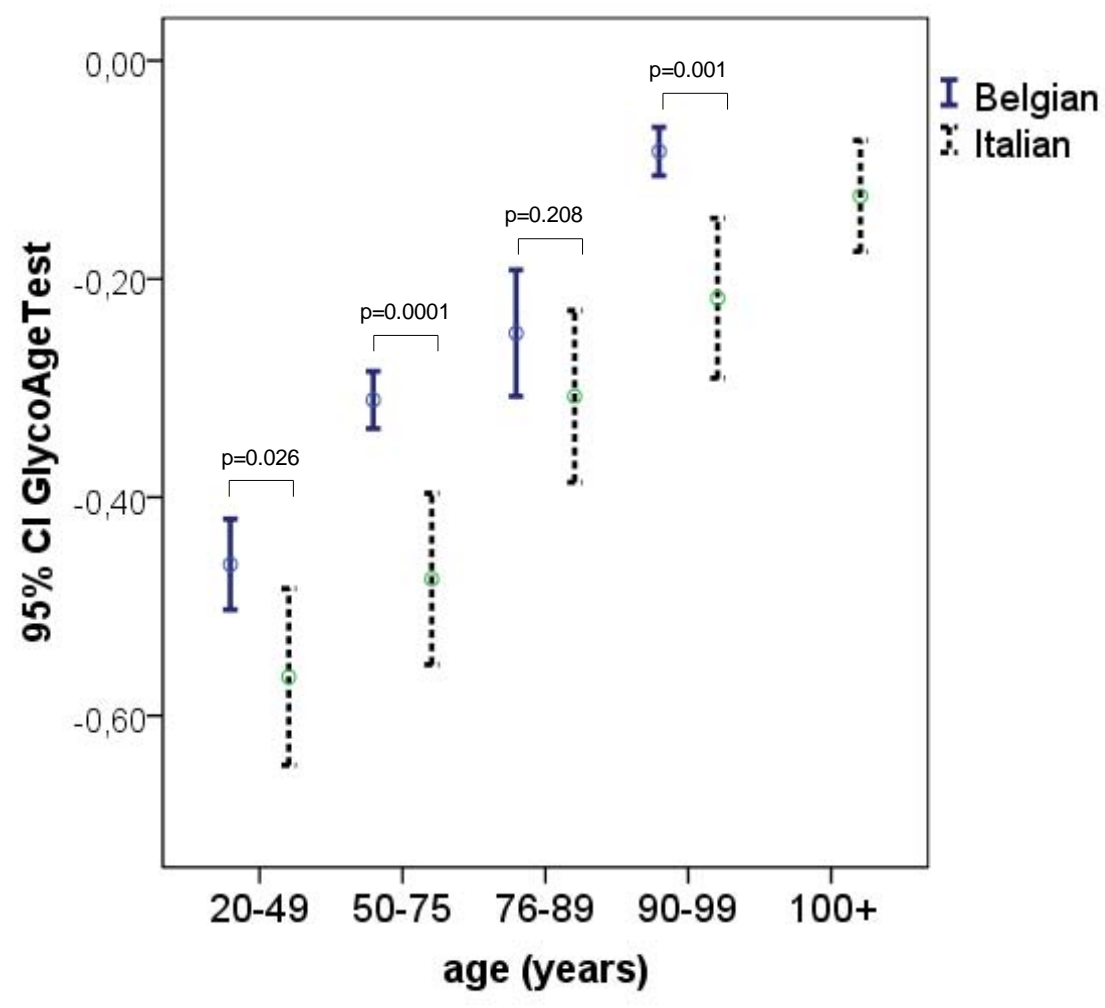

Figure 4 
Vanhooren et al. Serum N-glycan biomarker

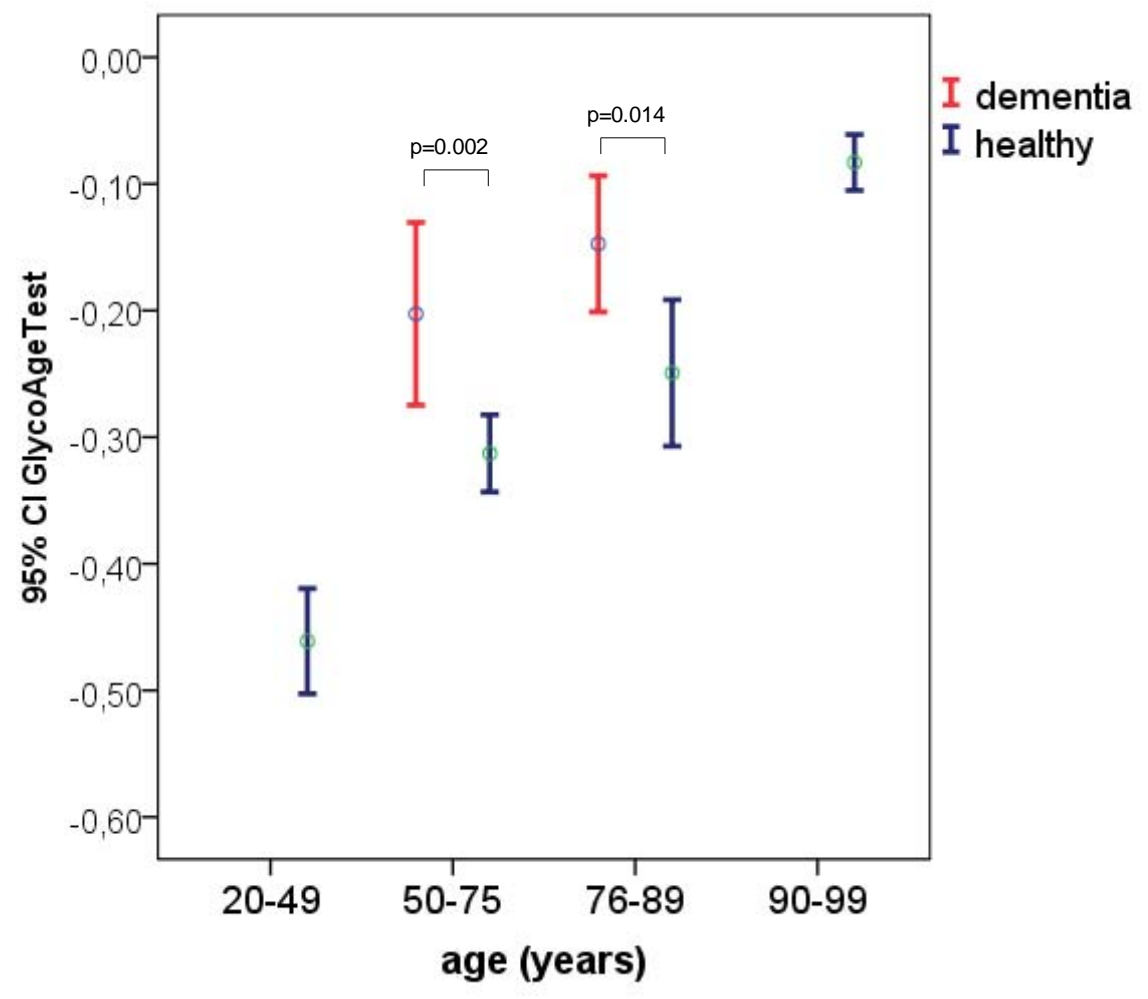

Figure5 
Vanhooren et al. Serum N-glycan biomarker

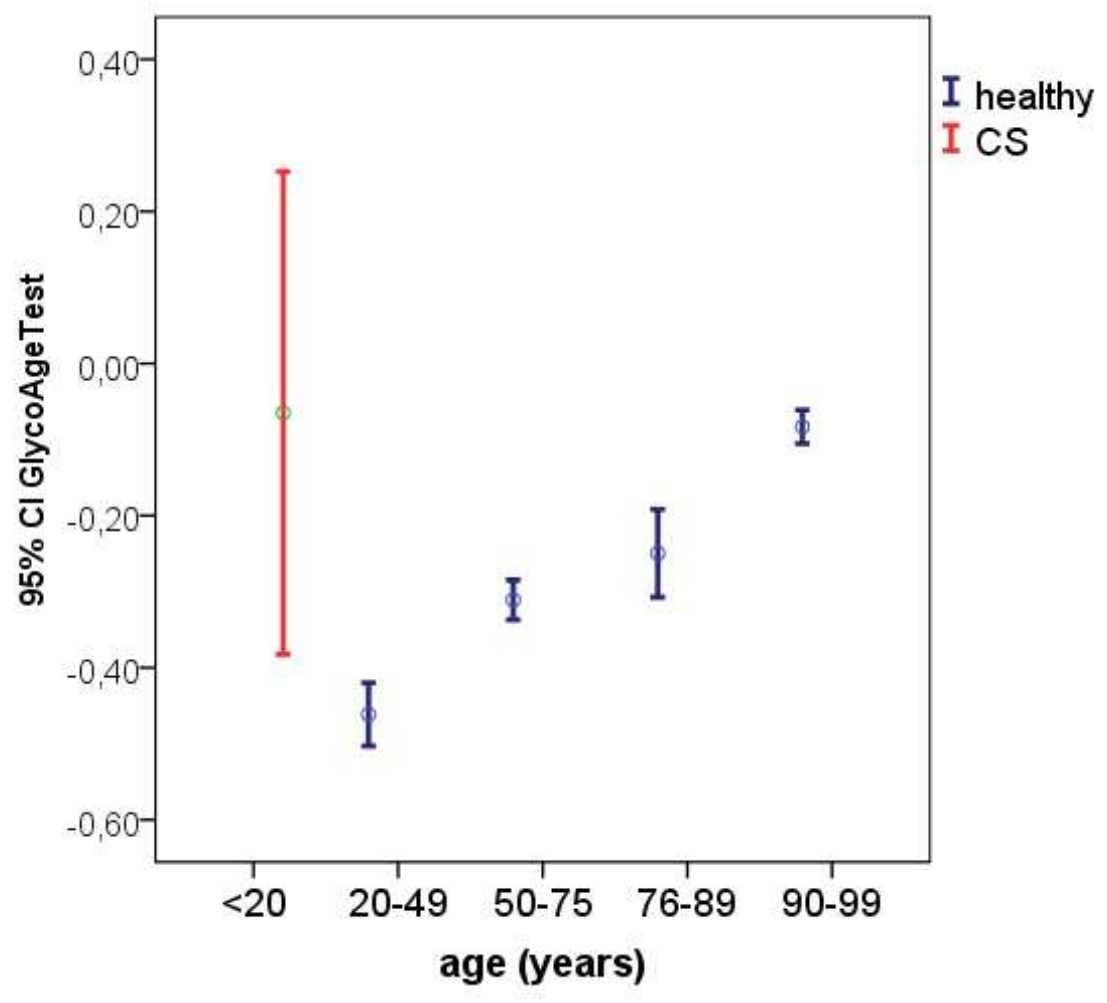

Figure 6 